\title{
Neuropathologic Studies of 'Epileptogenic' Tissue
}

\author{
Harry V. Vinters
}

\begin{abstract}
This mini-review will summarize some of the major contributions of Neuropathology to understanding the structural basis of several forms of intractable epilepsy. Because of space limitations, only selected morphologically defined brain abnormalities will be considered and illustrated. By definition, the types of specimen encountered by Neuropathologists are those resected to treat intractable seizure disorders. One can therefore infer that the 'lesions' encountered in some way caused or at least contributed to the epilepsy, though the leap from observed neuropathologic abnormalities to "seizuregenesis' is not always straightforward-indeed often is very tortuous and obscure. Nevertheless, a Neuropathologist working with a team of neurologists, neurosurgeons, electrophysiologists, neuropsychologists, and others, can contribute to a richer understanding of why seizures are triggered within human brain tissue, and therefore how they may be more effectively treated.
\end{abstract}

\begin{abstract}
RÉSUMÉ: Études neuropathologiques du tissu épileptogène. Cette mini-revue constitue un sommaire de certaines des contributions majeures de la neuropathologie à la compréhension des éléments structuraux de base de plusieurs types d'épilepsies pharmacorésistantes. Nous avons choisi de traiter et d'illustrer certaines anomalies cérébrales définies au point de vue morphologique, étant donné les contraintes d'espace. Par définition, les spécimens qu'examinent les neuropathologistes sont ceux qui ont été prélevés au cours du traitement chirurgical de l'épilepsie pharmacorésistante. On peut donc déduire que les « lésions » observées ont causé en quelque sorte ou ont pour le moins contribué à l'épilepsie, bien que le lien entre les anomalies neuropathologiques observées et la genèse des crises ne soit pas toujours direct mais souvent plutôt confus et obscur. Néanmoins, un neuropathologiste qui travaille avec une équipe de neurologues, de neurochirurgiens, d'électrophysiologistes, de neuropsychologues, ainsi qu'avec d'autres spécialistes, peut contribuer à une meilleure compréhension de la raison pour laquelle les crises sont déclenchées dans le tissu du cerveau humain et donc aux moyens par lesquels elles pourraient être traitées plus efficacement.
\end{abstract}

The surgical treatment of intractable epilepsy is now a widely accepted therapeutic modality. The University of Western Ontario (UWO) Epilepsy Surgery Programme, whose success was celebrated at this symposium in London, Ontario, has contributed greatly to that acceptance, and deserves hearty congratulations for this effort, which continues today. Indeed, as presentations during the celebratory day proved, the innovative UWO Epilepsy Programme moves from strength to strength.

One result of the growth of epilepsy surgery programs worldwide is that this innovative treatment provides a bounty of interesting lesions for Neuropathologists to examine and study. Unfortunately, since epilepsy surgery is most commonly carried out in medical centers with excellent neuroimaging and EEG facilities, together with expensive intraoperative support including anesthesia, this procedure is often not available to thousands of individuals who would benefit from it ${ }^{1}$. In the early days of therapeutic epilepsy surgery, Neuropathologists often struggled with the appropriate nomenclature for unique lesions that originated within epileptogenic brain - and to some extent this remains a problem to the present time. However, the meticulous approach to specimen analysis, exemplified by the early work of Gordon Mathieson at the Montreal Neurological
Institute (and many others) laid the foundations for our current approach to neuropathologic diagnosis ${ }^{2}$. Because corticectomy, lobectomy or even hemispherectomy for intractable seizures is often performed relatively early in the course of a seizure disorder (though never before a trial of therapeutic medications), histopathologic abnormalities are often observed in a relatively 'pristine' state, i.e. before secondary changes (e.g. hypoxicischemic injury, inflammation) related to seizures themselves complicate the morphologic picture. In large registries of specimens from epilepsy surgeries, e.g. the European Epilepsy Brain Bank, the most common type of specimen to be seen by the Neuropathologist is hippocampal sclerosis [HS]-

\footnotetext{
From the David Geffen School of Medicine at UCLA, Section of Neuropathology, Ronald Reagan-UCLA Medical Center, Los Angeles, California, USA. Received January 26, 2012. Final Revisions Submitted August 7, 2012. Correspondence to: Harry V. Vinters, David Geffen School of Medicine at UCLA, Section of Neuropathology, Ronald Reagan-UCLA Medical Center, 650 Charles Young Drive South, CHS room 18-170, Los Angeles, California, 90095-1732, USA.

Email: hvinters@mednet.ucla.edu.
} 
constituting over one third of all cases ${ }^{3}$. Less commonly encountered lesions include longterm epilepsy associated tumors [LEATs], which constitute approx. $25 \%$ of specimens, followed by malformations of cortical development [MCDs, 15.5\%], then abnormalities described as 'vascular' (arteriovenous malformations, cavernous hemangiomas), glial scars and encephalitis (including Rasmussen encephalitis, see below). Clinicopathologic data from such registries also reveal the lengthy intervals between seizure onset and operative intervention-which can range from 8.7 years (for encephalitis) to $22+$ years (for HS).

How should tissue from epilepsy surgery be handled? This will depend upon the diagnostic and research interests of a given institution. Initially, clinicians will want to know the answer to the question: what is the lesion? In order to answer this, the Neuropathologist can obtain significant information (and often a definitive diagnosis) from examining routine hematoxylin and eosin (H\&E)-stained sections of the resected tissue. For instance, the diagnoses of severe focal cortical dysplasia (FCD, ILAE type IIB), Rasmussen encephalitis, or an (old) ischemic lesion or scar can be made quite accurately without benefit of special stains. However, special and immunohistochemical stains are of value in studying various lesions. The Kluver-Barrera stain highlights neuronal cytoarchitectural features and allows for a more detailed assessment of cortical organization (and disorganization), facilitating the diagnosis of more subtle degrees of $\mathrm{FCD}^{4}$. Immunohistochemistry using primary antibodies to GFAP highlights reactive astrocytic proliferation, and anti-CD68 and Iba-1+ demonstrate macrophages and microglial cells, thus are especially useful in highlighting the gliotic/chronic inflammatory components of structural or neoplastic lesions (e.g. FCD, LEATs) and chronic encephalitis, e.g. of the type seen in patients with Rasmussen encephalitis (RE) $)^{5,6}$. Cytoskeletal (neuronal) abnormalities (e.g. in severe FCD) may be detected or accentuated using primary antibodies to various neurofilament epitopes $^{7}$, while anti-synaptophysin can highlight synaptic abnormalities within dysplastic neocortex, as well as neuronal heterotopias within the subcortical white matter. Increasingly, $\mathrm{NeuN}$ is recognized as a very useful marker to demonstrate neurons, thus allowing foci of malformed cortex to be distinguished from normal cortical ribbon ${ }^{8}$. Many of these immunohistochemical approaches can be used to quantify neuronal density, size, distribution and configuration within epileptogenic foci.

An answer to the question 'what caused the lesion' is more problematic for the Neuropathologist. For instance the etiology of Rasmussen encephalitis, a unihemispheric progressive inflammatory and destructive disorder associated with epilepsia partialis continua and hemiparesis, has been sought since its initial discovery in $1958^{9}$ - thus far without convincing proof as to its cause $\mathrm{e}^{10,11}$. However, in order to pursue sophisticated molecular, neurochemical and other biological studies of resected tissue, it is useful for the Neuropathologist (with appropriate Institutional Review Board approval) to freeze portions of the freshly removed tissue, that can then be analyzed further. Perhaps the most important question in examining tissue from epilepsy surgeries is: How was the lesion producing seizures? In order to optimally address this, close collaboration is required between the 'tissue handler' and a committed neurophysiologist who can further study the electrophysiological properties of select neuroglial elements within a corticetomy specimen, culminating in injection of a 'recorded cell' with a dye that can then be used to identify the cells in a tissue section ${ }^{12,13}$. Again, in order to achieve this, close cooperation is required between the Neuropathologist and her/his colleagues having electrophysiological expertise, especially because the tissue needs to be transported to the electrophysiology laboratory as rapidly as possible in order to optimize experimental results..

\section{Table: Structural Lesions Associated with Intractable Epilepsy}

Ammon's horn (hippocampal) sclerosis (AHS, HS, mesial temporal sclerosis, MTS )

Malformative:

--Parenchymal-malformations of cortical development, cortical dysplasia, neuronal migration disorders

--Vascular---arteriovenous malformation, cavernous hemangioma

Neoplasms: especially longstanding/longterm epilepsy-associated turmors (LEATs)

Familial/genetic \& Metabolic disorders:

--With focal lesions (neurocutaneous disorders, phakomatoses)-Tuberous sclerosis complex (TSC), neurofibromatosis,

Sturge-Weber-Dimitri syndrome (S-W-D is sporadic but often grouped with phakomatoses)

--With diffuse lesions---Lysosomal enzyme deficiencies, peroxisomal disorders, mitochondrial 'cytopathies', Lafora body disease (myoclonus), myoclonic eplepsies, others (e.g. Alexander's disease, ceroid lipofuscinosis)

Cerebrovascular disease and trauma:

--Ischemic, hemorrhagic, post-traumatic scars

Inflammatory/infectious:

--Fulminant encephalitis (e.g. due to Herpes simplex, other viruses), chronic (e.g. parasitic infestations such as cysticercosis)

--Rasmussen (pathogen-free) encephalitis 
The modern era of epilepsy surgery has coincided with the recognition of the importance of malformations of cortical development (MCDs) as an important cause of intractable epilepsy in infants and children, less commonly in adults, 4,7,8,14-16. The initial description of this entity is attributed to Prof. David Taylor ${ }^{17}$; however, it is of interest that for several years after Taylor's elegant clinicopathologic description of 'focal cortical dysplasia/FCD', it hardly received a mention in the epilepsy literature. That has changed drastically within the past 10-15 years, so that currently almost 100 (peer-reviewed) publications annually appear on this topic (G.W. Mathern, personal communication). When one considers surgical resection specimens from individuals 18 years-of-age or younger, cortical dysplasia is by far the most common lesion identified, followed by LEATs as a distant second. The younger the age of surgery for a child or infant, the greater is the likelihood that the resected tissue will show FCD. Various terms have been utilized to describe this entity-more correctly, group or range of entities: these include MCD, FCD, neuronal migration disorders, and synaptic dysgenesis.

Malformations of cortical development can cause a variety of seizure types, including infantile spasms (ISS), which may onset within days or weeks of the infant's birth. While it would appear counterintuitive to imagine a neurosurgical treatment to be of value for a malformation of the cerebral cortical mantle, high resolution neuroimaging and electrophysiological localization techniques have shown that MCDs can be rather welllocalized - to one cerebral hemisphere, one or two lobes of a hemisphere, a single lobe, or even one region of cortex confined to a single lobe-making them highly amenable to therapeutic resection. The extent of the resection is obviously tailored to the extent of the cortical abnormality, and assumes that the abnormality is confined to one cerebral hemisphere. A focal cortical lesion can be resected by removing a few $\mathrm{cm}$ of lesional tissue, whereas a hemispheric abnormality (causing, in the extreme case, hemimegalencephaly/ HME) may necessitate a hemispherectomy, either anatomical or modified ${ }^{18,19}$.

The terminology used to describe neuropathologic features of FCD (sometimes abbreviated as CD) has undergone several iterations over the past $15-20$ years $^{4,20}$. Most recently, a Task Force of the International League Against Epilepsy has formulated diagnostic criteria by which the severity or 'grade' of FCD can be assessed-hopefully reproducibly among various Centers $^{8}$. The interested reader is referred to the detailed article summarizing the rationale for this classification system. However, a few points are worth emphasizing. Immunohistochemical stains (especially using primary antibodies to NeuN) are deemed to be highly useful in identifying subtle degrees of FCD. Focal cortical dysplasia type I represents a fairly subtle degree of neuronal disorganization within a region of cortex, identified as abnormal radial cortical lamination (type Ia), abnormal tangential cortical lamination (type Ib), or a combination of Ia and Ib (which then becomes type Ic). Focal cortical dysplasia type II is subdivided into lesions that contain dysmorphic neurons (IIa) or additionally contain 'balloon cells' (type IIb) (see Figure 1). Of note, even the changes of severe (IIb) FCD can, within a given corticectomy specimen, be patchy and focal, requiring extensive sampling of

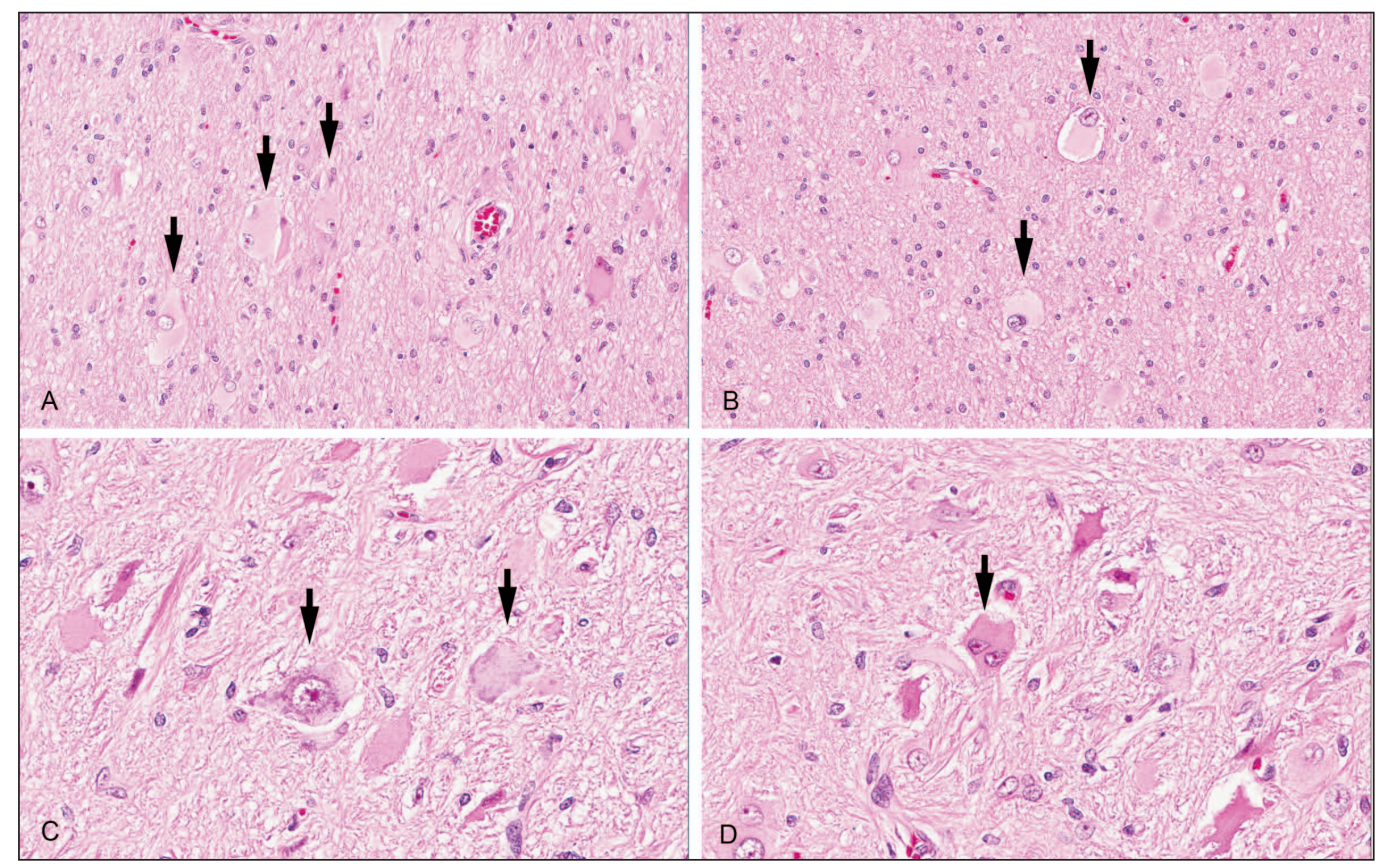

Figure 1: Focal cortical dysplasia, ILAE type IIb. (All sections are from the same specimen, and all micrographs are of $H \& E$-stained sections.) A,B. Characteristic 'balloon cells'(arrows), with glassy eosinophilic cytoplasm and eccentric nuclei showing coarse chromatin, with variably sized nucleoli. C,D. Dysmorphic neurons (arrows) with coarse disorganized Nissl substance and abnormal shapes. Arrow in ' $D$ ' illustrates a binucleate cell that probably represents a neuron. (Original magnification panels $A, B X 20$, panels $C, D X 40$ ). 

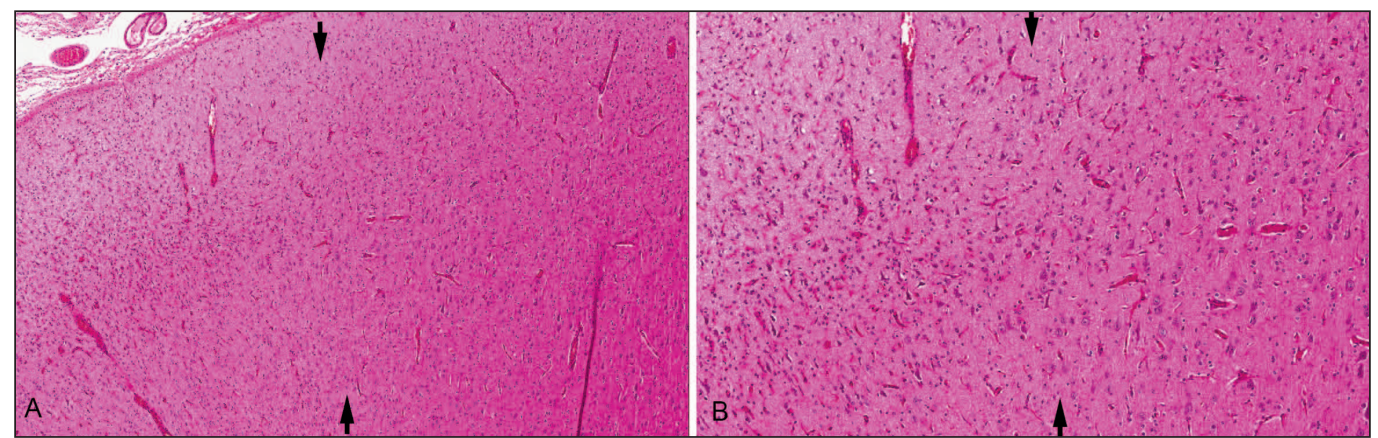

Figure 2: Rasmussen encephalitis. H\&E-stained sections show the relatively 'sharp' boundary between affected cortex (indicated by arrows) at left and more normal cortex at right. (Original magnification A X 4.0, B X 8.0).

a given specimen and careful evaluation of the resultant histologic sections; even so, the diagnosis of FCD can usually be made on a routine $\mathrm{H} \& \mathrm{E}-$ stained histologic section. Focal cortical dysplasia type III is used to describe FCD seen in association with a 'principal lesion' (e.g. HS, a glioneuronal tumor, or a vascular malformation). As might be expected, the most 'dramatic' lesion, i.e. FCD type IIb, is the easiest to be reproducibly identified among different laboratories and Neuropathologists. However, with the advent of new approaches to evaluating neuronal layering within cerebral cortex using 'layer-specific probes' and quantitative assessment of neuronal density and distribution, more subtle degrees of FCD may be routinely discovered ${ }^{21}$.
The etiology of MCDs/FCDs is unclear and animal models are not consistently useful in providing data that suggest its molecular/cellular/neurobiologic origin. Our group has proposed the "Dysmature cerebral developmental hypothesis" as one explanation for $\mathrm{CD}^{22}$. Numerous investigators have highlighted the similarity of FCD type IIb to the cortical tubers (often epileptogenic) found within brains of tuberous sclerosis complex (TSC) patients, suggesting molecular (and possibly even genetic) links between the two disorders ${ }^{7,8,14,15}$. Insulin signaling pathways, which affect neuronal morphology and size, differ substantively between TSC tubers and FCD lesions, largely as the result of mutations in the TSC1 or TSC2 genes in TSC patients $^{23}$. A recent animal model of TSC neuropathologic

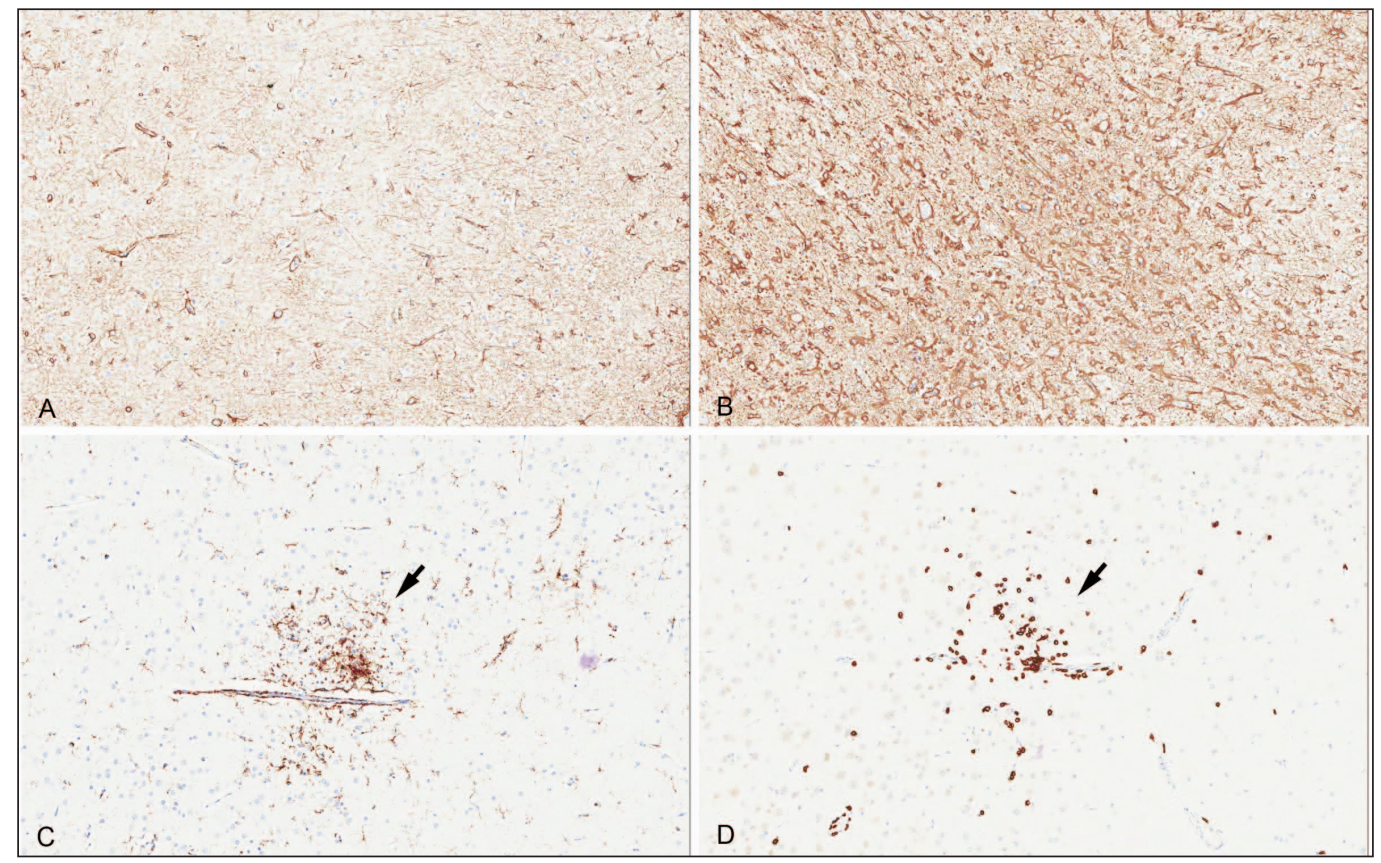

Figure 3: Rasmussen encephalitis. All micrographs are from the same specimen. A. GFAP immunostained section from relatively 'normal' cortex, showing rare immuno-reactive astrocytes. B. A prominently affected region of cortex (GFAP-immunostained section), showing dense astrocytic gliosis. C. Microglial nodule (arrow) adjacent to a capillary (cut in longitudinal section) highlighted by CD68 immunohistochemistry. D. Collection of CD3immunoreactive T-cells (arrow). (All panels from micrographs with original magnification of 10X) 
change will almost certainly provide insights into the relationship between the two types of cortical lesion ${ }^{24}$.

Rasmussen encephalitis ( $\boldsymbol{R} \boldsymbol{E})$, first described in 1958, is another enigmatic entity that causes epilepsia partialis continua (EPC), unihemispheric atrophy, and hemiparesis, as the result of severe chronic inflammation almost always confined to one cerebral hemisphere ${ }^{9}$; indeed, the initial clinicopathologic description by Theodore Rasmussen et al can hardly be improved upon. The neuropathologic features of RE include multifocal chronic inflammation within cerebral cortex, characterized by infiltraton of T-lymphocytes and extensive proliferation of microglia ${ }^{25}$; the lesions are surprisingly well demarcated from morphologically normal cerebral cortex, often a few um away from affected brain (Figure 2). Microscopically, the 'injured' tissue eventually becomes markedly gliotic, and contains both activated microglia (often in the form of microglial nodules) and dense astrocytosis (Figure 3). Despite the resemblance of this constellation of neuropathologic abnormalities to "viral encephalitis', no etiologic agent has consistently been demonstrated within RE brain ${ }^{10,11}$. Discussions at a recent symposium/workshop on RE, involving experts on inflammatory, infectious and autoimmune CNS disorders, centered on whether RE is a brain infection resulting from an as yet undiscovered/unrecognized (probably viral) agent, an anomalous CNS response to a ubiquitous agent, or an autoimmune disease. Recently, interesting vascular changes have been described in resected RE cortical specimens, especially in the second of 'staged' operations for this disorder ${ }^{26}$.

While LEATs are a clinically and diagnostically challenging group of entities (from both neuropathologic and management perspectives), they will not be considered here; the reader is referred to an excellent recent review ${ }^{27}$.

\section{CONCLUSION}

The availability of brain tissues from epilepsy surgeries presents a remarkable opportunity for Neuropathologists to 'facilitate' discovery of lesions that may be of major importance in causing focal or generalized seizure disorders-including their etiology. This involves coordinating the activities of a multi-disciplinary group of investigators with various types of expertise-but the potential rewards are immense.

\section{REFERENCES}

1. Engel J. Current concepts - surgery for seizures. N Engl J Med. 1996;334:647-52.

2. Mathieson G. Pathologic aspects of epilepsy with special reference to the surgical pathology of focal cerebral seizures. Adv Neurol. 1975;8:107-38

3. Blumcke I, Spreafico R. Cause matters: A neuropathological challenge to human epilepsies. Brain Pathol. 2012;22:347-9.

4. Mischel PS, Nguyen LP, Vinters HV. Cerebral cortical dysplasia associated with pediatric epilepsy. Review of neuropathologic features and proposal for a grading system. J Neuropathol Exp Neurol. 1995;54:137-53.

5. Sofroniew MV, Vinters HV. Astrocytes: Biology and pathology. Acta Neuropathol. 119:7-35.

6. Wirenfeldt M, Babcock AA, Vinters HV. Microglia-insights into immune system structure, function, and reactivity in the central nervous system. Histol Histopathol. 2011; 26:519-30.

7. Crino PB, Miyata H, Vinters HV. Neurodevelopmental disorders as a cause of seizures: neuropathologic, genetic, and mechanistic considerations. Brain Pathol. 2002;212-33.
8. Blumcke I, Thom M, Aronica E, Armstrong DD, Vinters HV, et al. The clinicopathologic spectrum of focal cortical dysplasias: A consensus classification proposed by an ad hoc Task Force of the ILAE Diagnostic Methods Commission.

9. Rasmussen T, Olszewski J, Lloyd-Smith D. Focal seizures due to chronic localized encephalitis. Neurology. 1958; 8:435-45.

10. Vinters HV, Wang R, Wiley CA. Herpesviruses in chronic encephalitis associated with intractable childhood epilepsy. Hum Pathol. 1993;24:871-9.

11. Farrell MA, Droogan O, Secor DL, Poukens V, Quinn B, Vinters HV. Chronic encephalitis associated with epilepsy: Immunohistochemical and ultrastructural studies. Acta Neuropathol. 1995;89:313-21.

12. Cepeda C, Hurst RS, Flores-Hernandez J, Hernandez-Echeagaray E, et al. Morphological and electrophysiological characterization of abnormal cell types in pediatric cortical dysplasia. J Neurosci Res. 2003;72:472-86.

13. Cepeda C, Andre VM, Yamazaki I, Hauptman JS, et al. Comparative study of cellular and synaptic abnormalities in brain tissue samples from pediatric tuberous sclerosis complex and cortical dysplasia type II. Epilepsia. 2010; 51 (suppl. 3): 160-165.

14. Farrell MA, DeRosa MJ, Curran JG, Secor DL, et al. Neuropathologic findings in cortical resections (including hemispherectomies) performed for the treatment of intractable childhood epilepsy. Acta Neuropathol. 1992;83:246-59.

15. Vinters HV, Fisher RS, Cornford ME, Mah V, et al. Morphological substrates of infantile spasms: Studies based on surgically resected cerebral tissue. Child's Nerv Sys. 1992; 8:8-17.

16. Aronica E, Becker AJ, Spreafico R. Malformations of cortical development. Brain Pathol. 2012; 22:380-401.

17. Taylor DC, Falconer MA, Bruton CJ, Corsellis JA. Focal dysplasia of cerebral cortex in epilepsy. J Neurol Neurosurg Psychiatry. 1971;34:369-87.

18. Salamon N, Andres M, Chute DJ, Nguyen ST, et al. Contralateral hemimicrencephaly and clinical-pathological correlations in children with hemimegalencephaly. Brain. 2006;129 (Pt. 2): 352-65.

19. Cook SW, Nguyen ST, Hu B, Yudovin S, et al. Cerebral hemispherectomy in pediatric patients with epilepsy: comparison of three techniques by pathological substrate in 115 patients. J Neurosurg. 2004;100 (Suppl):125/141.

20. Palmini A, Najm I, Avanzini G, Babb T, et al.Terminology and classification of the cortical dysplasias. Neurology. 2004;62(6 Suppl 3):S2-8.

21. Hadjivassiliou G, Martinian L, Squier W, Blumcke I, Aronica E, Sisodiya SM, et al. The application of cortical layer markers in the evaluation of cortical dysplasia in epilepsy. Acta Neuropathol. 2010; 120:517-28.

22. Cepeda C, Andre VM, Levine MS, Salamon N, Miyata H, Vinters $\mathrm{HV}$, et al. Epileptogenesis in pediatric cortical dysplasia: the dysmature cerebral developmental hypothesis. Epilepsy Behav. 2006;9:219-35.

23. Miyata $\mathrm{H}$, Chiang AC, Vinters HV. Insulin signaling pathways in cortical dysplasia and TSC-tubers: tissue microarray analysis. Ann Neurol. 2004:56:510-19.

24. Goto J, Talos DM, Klein P, Qin W, Chekaluk YI, et al. Regulable neural progenitor-specific Tsc1 loss yields giant cells with organellar dysfunction in a model of tuberous sclerosis complex. Proc Natl Acad Sci USA. 108:E1070-9.

25. Wirenfeldt M, Clare R, Tung S, Bottini A, Mathern GW, Vinters $\mathrm{HV}$. Increased activation of Iba1+ microglia in pediatric epilepsy patients with Rasmussen's encephalitis compared with cortical dysplasia and tuberous sclerosis complex. Neurobiol Dis. 2009; 34:432-40.

26. Wagner AS, Yin NS, Tung S, Mathern GW, Vinters HV. Intimal thickening of meningeal arteries after serial corticectomies for Rasmussen encephalitis. Hum Pathol. 2012; 43:1308-13.

27. Thom M, Blumcke I, Aronica E. Long-term epilepsy-associated tumors. Brain Pathol. 2012;22:350-79. 